\title{
Dissemination and Sustainability of a Hospital-Wide Hand Hygiene Program Emphasizing Positive Reinforcement
}

\author{
Jeanmarie Mayer, MD; Barbara Mooney, RN, CIC; Adi Gundlapalli, MD, PhD; \\ Stephan Harbarth, MD, MS; Gregory J Stoddard, MPH; Michael A. Rubin, MD, PhD; \\ Louise Eutropius, RN, CIC; Britt Brinton, MT, CIC; Matthew H. Samore, MD
}

овјестіve. To increase and sustain hospital-wide compliance with hand hygiene through a long-term ongoing multidimensional improvement program emphasizing behavioral factors.

DESIGN. Quasi-experimental short study (August 2000-November 2001) and descriptive time series (April 2003-December 2006).

SETTING. A 450-bed teaching tertiary-care hospital.

InTERVENTIONS. An initial intervention bundle was introduced in pilot locations that addressed cognitive behavioral factors, which included access to alcohol sanitizer, education, and ongoing audit and feedback. The bundle was subsequently disseminated hospital-wide, along with a novel approach focused on behavior modification through positive reinforcement and annually changing incentives.

REsults. A total of 36,123 hand hygiene opportunities involving all categories of healthcare workers from 12 inpatient units were observed from October 2000 to October 2006. The rate of compliance with hand hygiene significantly improved after the intervention in 2 cohorts over the first year (from $40 \%$ to $64 \%$ of opportunities and from $34 \%$ to $49 \%$ of opportunities; $P<.001$, compared with the control group). Mean compliance rates ranged from $19 \%$ to $41 \%$ of 4174 opportunities (at baseline), increased to the highest levels of $73 \%-84 \%$ of 6,420 opportunities 2 years after hospital-wide dissemination, and remained improved at $59 \%-81 \%$ of 4,990 opportunities during year 6 of the program.

CONCLUSION. This interventional cohort study used a behavioral change approach and is one of the earliest and largest institution-wide programs promoting alcohol sanitizer from the United States that has shown significant and sustained improvements in hand hygiene compliance. This creative campaign used ongoing frequent audit and feedback with novel use of immediate positive reinforcement at an acceptable cost to the institution.

Infect Control Hosp Epidemiol 2011; 32(1):59-66

Hand hygiene can prevent healthcare-associated infections, ${ }^{1-3}$ and healthcare workers (HCWs) believe hand hygiene reduces the risk of infection. ${ }^{4,5}$ Evidence-based guidelines call on HCWs to practice hand hygiene to enhance patient safety. ${ }^{6-9}$ Nevertheless, there is a gap between evidence-based practice and actual adherence to hand hygiene, ${ }^{10}$ with an overall median compliance rate of $40 \%$ of opportunities. ${ }^{11}$

Multiple impediments to hand hygiene include inaccessibility of hand sanitizers, time constraints, forgetfulness, and lack of education and modeling. ${ }^{12,13}$ These barriers must be bridged for practice to improve. Interventions that address selected barriers have led to transient improvements in hand hygiene. ${ }^{14,15}$ Continued success likely calls for an ongoing multifaceted behavioral approach, ${ }^{10,11,16}$ as previously demonstrated by the first multidimensional, hospital-wide cam- paign to achieve sustained hand hygiene improvements in Europe. ${ }^{1}$

Social and behavioral models provide a theoretical framework to identify factors that affect behavior and construct interventions to improve healthcare practices. ${ }^{17,18}$ The theory of planned behavior, which has been used to understand and change hand hygiene behaviors, proposes that intention is the immediate determinant of behavior. ${ }^{19-22}$ Intention is predicted by intermediate variables, including attitude, the subjective norm, and perceived behavioral control, that can be addressed with interventions such as education, administrative support, and accessibility to needed tools. However, even when HCWs have good intentions and believe they perform good hand hygiene, actual practices may be suboptimal. ${ }^{21,23,24}$ Behavior modification using techniques such as positive reinforcement

From the University of Utah School of Medicine (J.M., A.G., G.J.S., M.A.R., M.H.S.), University of Utah Hospital (B.M., L.E., B.B.), and Salt Lake City VAMC IDEAS Center (M.A.R., M.H.S.), Salt Lake City, Utah; and University of Geneva Hospitals and Medical School, Geneva, Switzerland (S.H.).

Received May 4, 2010; accepted July 15, 2010; electronically published December 3, 2010.

(C) 2011 by The Society for Healthcare Epidemiology of America. All rights reserved. 0899-823X/2011/3201-0008\$15.00. DOI: 10.1086/657666 
has been successfully employed in patient care. ${ }^{25,26}$ However, relatively little has been published regarding the use of positive reinforcement in changing $\mathrm{HCW}$ hand hygiene behavior. ${ }^{27,28}$

We undertook a long-term, hospital-wide, multidimensional hand hygiene improvement program emphasizing behavioral factors as an enduring approach to increase hand hygiene compliance. The initial phase employed an intervention bundle focused on cognitive behavior determinants in pilot locations. This initial bundle was then disseminated hospital-wide during a second phase alongside additional interventions that used motivators and immediate positive reinforcement as a novel approach to modify behavior. Our main objective was to increase and sustain the hospital-wide rate of compliance with hand hygiene. Implementing a comprehensive ongoing program at a feasible cost was a secondary objective.

\section{METHODS}

\section{Setting and Participants}

The University of Utah Hospital (Salt Lake City, Utah) is a 450-bed teaching tertiary-care hospital. Over a 6-year study period (October 2000-October 2006), hand hygiene adherence was measured in 12 patient care units: 6 acute care units ( 322 beds), 1 oncology unit ( 25 beds), and 5 intensive care units (103 beds).

\section{Study Design}

To meet our objectives, 2 closely related studies were conducted. First, a prospective quasi-experimental cohort study that focused on cognitive behavioral factors was done in 3 groups of hospital locations, each comprising 2 patient units. Baseline data were collected for all 3 groups during period 1 (August-September 2000). A staggered introduction of an intervention bundle that targeted the intermediate behavioral variables from the theory of planned behavior was imple- mented in group A ( 2 units) during period 2 (October 2000November 2001) and in group B ( 2 units) during period 3 (May-November 2001), while group C ( 2 units) remained as a control group (Figure 1).

Second, a time-series design was used for hospital-wide dissemination of the hand hygiene campaign. Use of the initial intervention bundle was continued in groups $\mathrm{A}$ and $\mathrm{B}$ and introduced in group $C$ and in 4 of 6 additional units in group D during period 4 (April-December 2002) (Figure 1). A novel approach focusing on behavior modification through positive reinforcement and incentives was then added in all 12 hospital units starting in January 2003, during period 5.

\section{Interventions}

Initial intervention: social cognitive determinants of behavior. The first intervention was a bundle of components dealing with cognitive determinants of behavior. Attitudes towards hand hygiene were addressed by increasing knowledge through education: infection preventionists provided standardized unit in-service presentations, the hospital epidemiologist reviewed slide presentations with physician groups, and infection control personnel had one-on-one discussions with clinical staff while performing rounds. The subjective norm was communicated through ongoing audit with monthly feedback to make HCWs aware of their hand hygiene performance and of how that performance compared with the performance of others. Perceived behavioral control was enhanced by positioning dispensers of alcohol sanitizer in convenient locations; this provided HCWs with better tools to facilitate their hand hygiene.

Hospital-wide dissemination: behavior modification through positive reinforcement. The initial intervention was formally rolled out throughout the majority of remaining hospital locations during period 4 . Near the end of period 4 , a hand hygiene committee comprising infection preventionists, nurse managers from all hospital units, service directors, and the

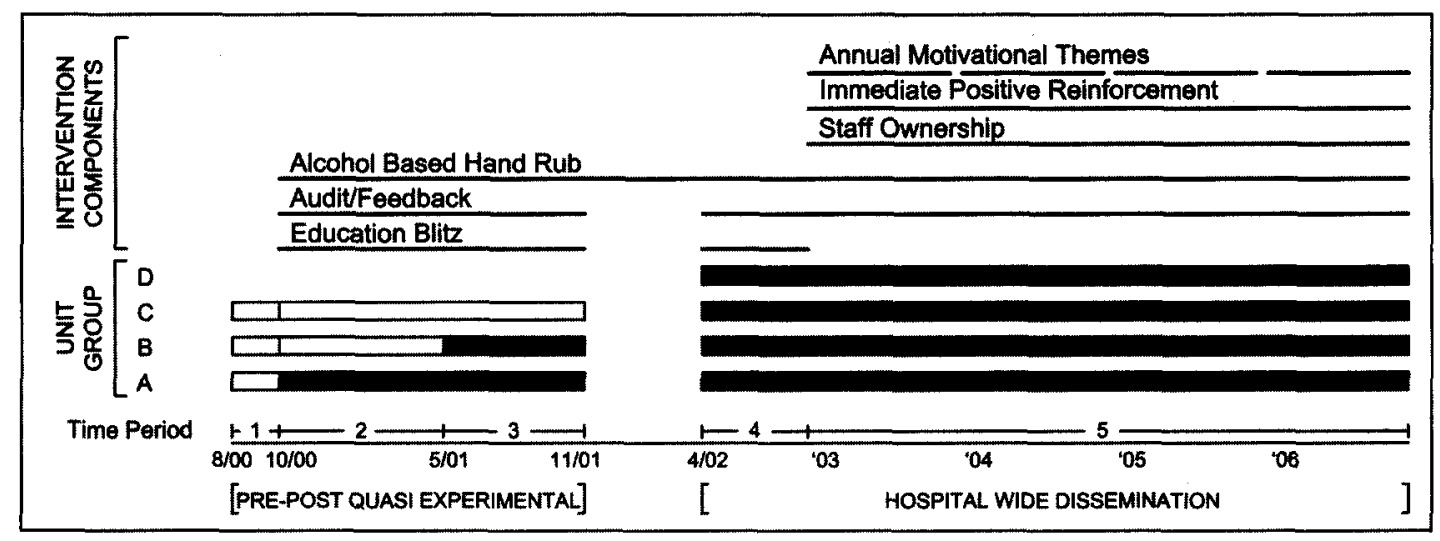

FIGURE 1. Overview of the hand hygiene campaign (August 2000-October 2006), with components of the intervention outlined across the top and groups of hospital unit locations undergoing hand hygiene observations across the bottom. Receipt of intervention by groups of hospital locations are indicated by gray bars. 
hospital epidemiologist was convened to encourage staff involvement and ownership. This group met monthly, and this meeting was a venue to publicly provide unit-specific feedback on hand hygiene compliance. Units with exemplary hand hygiene performance were rewarded with certificates and trophies that sparked friendly competitions. The hand hygiene committee discussed implementation barriers, shared success stories, and came up with ideas for catchy phrases, posters, and jingles. Subsequent strategies implemented during period 5 that focused on behavior modification originated from the Infection Control department and the hand hygiene committee. These positive reinforcements and motivators were revised annually to keep motivation high by providing new themes and mottoes. A popular and lasting immediate positive reinforcement was the "Caught in the Act" chocolate bar, which was handed out on the spot to HCWs "caught" performing hand hygiene. Early in 2003, chocolates were distributed by a weekly roving Infection Control "candy bar patrol" that consisted of an infection preventionist and a physician epidemiologist. The role of giving positive rewards was later transitioned to the unit managers, or "the bosses," to enhance unit ownership and impact for recipients. Each year, the hand hygiene committee generated new motivational campaign themes to maintain interest. An example of a group motivator theme was the "War on Germs" to encourage unit teamwork. Catchy posters were placed throughout the hospital calling unit "troops" to battle and publicizing that the unit with the best hand hygiene compliance would win a pizza party as the "spoils of war." "Rub it in to Win" was an individual incentives theme, in which individuals who were caught in the act of performing hand hygiene were entered into monthly drawings to win prizes.

\section{Definition of Hand Hygiene Opportunity and Compliance}

Hand hygiene was monitored using standardized, locally developed instruments including an instruction sheet and audit form. A usable opportunity for hand hygiene was defined as an observation in which an HCW was seen touching a patient or patient environment. An observation could be prolonged until completion of a patient care episode with either hand hygiene, the touching of a different patient, or the touching of something outside the patient's environment. Hand hygiene was defined as either washing hands with soap and water or cleaning hands with an alcohol sanitizer. Hand hygiene adherence was assessed during a patient care episode and was evaluated before, after, or before and after HCW contact with a patient or patient's environment.

\section{Observer Assessment of Hand Hygiene Outcome Measurements}

Nine part-time employees were trained as hand hygiene monitors over the 6-year study period and received training from an experienced mentor using a printed structured protocol. When no discrepancies between trainees and mentor were identified, training was considered to be complete. Inter-observer variability was formally evaluated during 7 sessions in which 2 monitors evaluated the same hand hygiene opportunity. For 87 observations, inter-rater reliability was good (mean $\kappa, 0.78$; range, 0.65-0.91).

Monitoring was ongoing throughout the campaign. Monitors spent an average of 10-14 hours per week collecting and recording observations, and although they spent less time on individual units as additional locations were added, a minimum of 30 usable observations per unit per month were required. Monitors randomly selected the order in which the units were evaluated each week. Observations were collected over all shifts and days of the week. Monitors positioned themselves unobtrusively outside rooms in locations from which they could view patient care interactions. Immediate feedback was not provided.

\section{Survey}

A self-administered survey assessing attitudes toward hand hygiene and exposure to the program was collected as a crosssectional convenience sample near the end of period 5 in March 2005. The survey consisted of 9 questions in 5 content areas. Attitudes toward hand hygiene and receipt of positive reinforcement were assessed using true/false questions. A 4point Likert scale measured the frequency of exposure to interventions and personal participation in reinforcing hand hygiene.

Surveys were distributed to staff on clinical units. Participation was voluntary and anonymous, but participants were asked to report their HCW category and hospital location. Completed surveys were returned in a folder and collected by the Infection Prevention department.

\section{Infection Prevention Practices and Infection Rates}

Surveillance cultures for multidrug-resistant organisms were not routinely performed over the course of the hand hygiene program, and no special contact isolations were implemented. Rates of hospital-associated infection with methicillin-resistant Staphylococcus aureus (MRSA) or vancomycin-resistant enterococcus (VRE) were calculated using an objective proxy measure (the number of first-time case patients during a 12month period from whom a culture sample positive for MRSA or VRE was collected $>2$ days after hospital admission or within 30 days after a previous hospitalization per 100 patient hospital admissions).

\section{Statistical Analysis}

The statistical analysis conformed to the designed staggered introduction of the intervention. In the first comparison, group A crossed over from not receiving the intervention to receiving it, whereas group $B$ remained without the intervention during the same time (periods 1 and 2 ). To verify that improved hand hygiene compliance was attributable to the intervention (and not to background "period effect" 
TABLE 1. Rates of Hand Hygiene Compliance during Varying Time Periods of the Campaign for Groups of Hospital Unit Locations

\begin{tabular}{|c|c|c|c|c|c|}
\hline \multirow[b]{2}{*}{ Variable } & \multicolumn{3}{|c|}{ Quasi-experimental phase } & \multicolumn{2}{|c|}{ Hospital dissemination phase } \\
\hline & $\begin{array}{c}\text { Period 1: } \\
\text { Aug-Sep 2000 }\end{array}$ & $\begin{array}{c}\text { Period 2: } \\
\text { Oct 2000-Apr } 2001\end{array}$ & $\begin{array}{c}\text { Period 3: } \\
\text { May-Oct 2001 }\end{array}$ & $\begin{array}{c}\text { Period 4: } \\
\text { Apr-Dec } 2002 \\
\end{array}$ & $\begin{array}{l}\text { Period } 5^{a}: \\
2003-2006\end{array}$ \\
\hline \multicolumn{6}{|l|}{ Group A (2 units) } \\
\hline Intervention & No & Yes & Yes & Yes & Yes \\
\hline No. of observations & 529 & 1,775 & 695 & 846 & 4,985 \\
\hline Compliance rate, $\%$ of opportunities & 40 & $64^{\mathrm{b}}$ & 66 & 68 & 81 \\
\hline Intervention & No & No & Yes & Yes & Yes \\
\hline No. of observations & 507 & 1,450 & 659 & 777 & 4,020 \\
\hline Compliance rate, $\%$ of opportunities & 27 & 34 & $49^{c}$ & 46 & 77 \\
\hline \multicolumn{6}{|l|}{ Group C (2 units) } \\
\hline Intervention & No & No & No & Yes & Yes \\
\hline No. of observations & 225 & 1,120 & 343 & 788 & 3,823 \\
\hline Compliance rate, $\%$ of opportunities & $\ldots$ & $\ldots$ & $\ldots$ & 36 & 72 \\
\hline
\end{tabular}

NOTE. $P$ values for the "period times hospital group" interaction term were determined from a mixed effects logistic regression model controlling for healthcare worker type and accounting for observations clustered within unit. NA, not applicable.

a Period 5 aggregates 4 years of hand hygiene measurements. The sustainability of the intervention for all cohorts is reported descriptively, because a control cohort was not available.

b $P<.001$ for comparison of group $A$ to group $B$ on the period 1 to period 2 change.

' $P<.001$ for comparison of group B to group $C$ on the period 2 to period 3 change.

caused by attitudes changing over time independently of the intervention), a second comparison was provided by the study design: group B crossed over from not receiving the intervention to receiving it, whereas group $C$ remained without the intervention during the same time (periods 2 and 3). For each of these 2 comparison opportunities, data were modeled using a mixed effects logistic regression model to account for lack of independence in the observations caused by clustering. Three levels were specified in the model: observations were nested within HCW type, then nested within hospital unit. Period and intervention were included as 2 indicator variables, along with a "period times intervention" interaction term. The test of the hypothesis that the intervention improved hand hygiene compliance was provided by the Wald test for the "period times intervention" interaction term.

The sustainability of the intervention for all cohorts (20032006) is reported descriptively, because a hypothesis test would be unable to rule out a period effect without a control cohort. Survey results based on a 4-point Likert scale were recorded to a dichotomous scale to simplify presentation. Fisher's exact test was used to compare survey responses by HCW category. Stata software, version 11.0 (Stata), was used for all statistical analyses.

\section{RESULTS}

\section{Characteristics and Overview of the Study}

A total of 36,123 hand hygiene opportunities involving all categories of HCWs from 12 inpatient units were observed from October 2000 through October 2006. Observations were distributed as follows: at baseline, there were $4,174(12 \%)$; at initial intervention, there were $6,354(18 \%)$; in 2003 , there were $8,679(24 \%)$; in 2004 , there were $6,420(18 \%)$; in 2005 , there were 5,376 (15\%); and in 2006, there were 4,990 (14\%). Most observations were of nursing staff $(28,868[80 \%])$, followed by physicians $(4,328[12 \%])$, and other HCW categories $(2,927[8 \%])$.

HCWs rapidly accepted alcohol sanitizer in place of soap and water, with use of sanitizer accounting for an increasing proportion of overall hand hygiene cleaning actions, as follows: $7 \%$ at baseline; $55 \%$ during the initial intervention bundle; $76 \%$ in 2003 ; and $\geqslant 85 \%$ in 2004 and thereafter.

\section{Hand Hygiene Compliance}

Hand hygiene compliance rates over the course of the study are provided in Table 1 . When controlling for HCW type and unit as random effects in the mixed-effects logistic regression model, we found that group A had a statistically significant increase in the rate of hand hygiene compliance from before introduction of the initial intervention bundle (period 1) to after the initial intervention (period 2) $(P<.001)$, whereas group $B$, acting as a control group, did not show a significant increase. Similarly, when group B was exposed to the initial intervention bundle, the compliance rate increased significantly from before the intervention (period 2) to after the intervention (period 3) $(P<.001)$, whereas group $\mathrm{C}$, as the new control group, did not show a significant change in the 


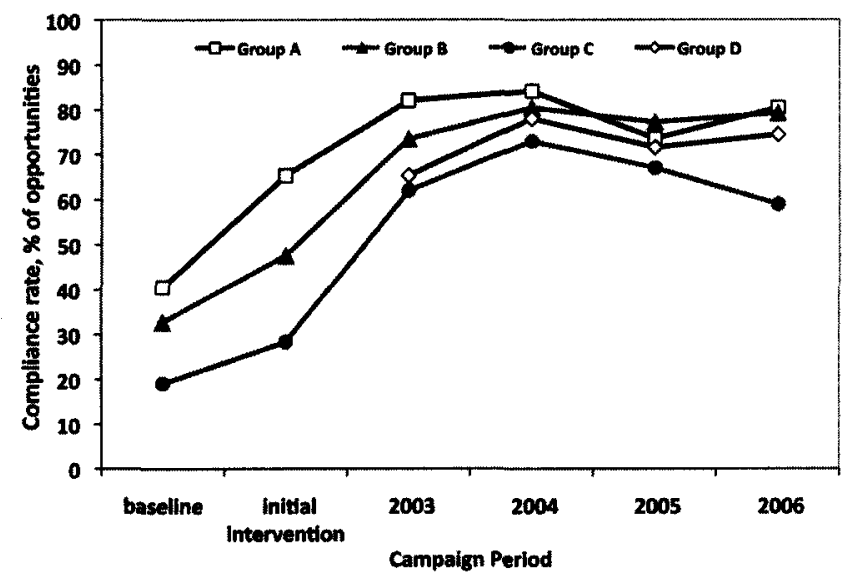

FIGURE 2. Rates of compliance with hand hygiene over the 6year campaign across all 4 groups of hospital unit locations. Group A (open squares and solid black line), group B (filled triangles and dashed black line), and group C (filled circles and dashed black line) had baseline hand hygiene adherence measured, whereas the remainder of unit locations in group D (open diamonds and solid black line) had hand hygiene adherence measured only during the hospitalwide dissemination of the motivational campaign.

compliance rate. Table 1 shows an improvement in the compliance rate was sustained in all groups when the hand hygiene program was disseminated hospital-wide, with larger increases seen in groups $C$ and $D$, for whom first exposure to the initial intervention bundle occurred in period 4 .

The hand hygiene compliance rate peaked in all hospital locations 2 years after hospital-wide dissemination in 2004 (Figure 2). The compliance rate decreased in 2005 but began to increase in 3 of the 4 groups 4 years after hospital-wide dissemination and 6 years after initial intervention. The mean compliance rate ranged from $19 \%$ to $41 \%$ of 4,174 opportunities at baseline, increased to the highest levels of $73 \%-$ $84 \%$ of 6,420 opportunities in 2004, and remained improved at $59 \%-81 \%$ of 4,990 opportunities in 2006 .

The average compliance rate differed by HCW category; nurses and other HCWs practiced better hand hygiene than did physicians in all study periods (Table 2). At baseline, the mean hand hygiene compliance rate was $27 \%$ of 3,173 observations for nursing staff, $35 \%$ of 487 observations for other ancillary HCWs, and $27 \%$ of 516 observations for physicians. By the end of the study, the greatest increase in hand hygiene compliance rate had occurred among nursing staff (48\% increase from baseline), and the smallest increase had occurred among physicians (33\% increase from baseline).

\section{Survey Results}

The response rate for distributed surveys was $63 \%$ (169 of 270 surveys returned) (Table 3 ). The attitude towards hand hygiene did not differ significantly between nursing staff and physicians, although nurses reported having more exposure to program incentives (Table 3). The majority believed hand hygiene was very important, and both groups reported that they could improve hand hygiene practices. Nursing staff were more likely to receive praise and awards in the presence of peers. Nurses reported receiving feedback on performance more frequently than did physicians, and more nurses reported that an authority figure regularly reminded them to practice hand hygiene. Nurses discussed hand hygiene frequently with coworkers; neither group regularly reminded others to perform hand hygiene.

\section{Rates of Infection with Multidrug-Resistant Organisms}

During the hand hygiene campaign, the mean annual incident rate of hospital-associated MRSA infection, as measured by clinical culture results and expressed as the number of cases per 100 patient admissions to the hospital, was 0.313 in 2000; 0.635 in 2001; 0.808 in 2002; 0.700 in 2003; 0.431 in 2004; 0.468 in 2005; and 0.476 in 2006. During this same time, the mean annual incident rate of hospital-associated VRE infection, as measured by clinical culture results and expressed as the number of cases per 100 patient admissions to the hospital, were 0.210 in 2000; 0.214 in 2001; 0.204 in 2002; 0.214 in 2003; 0.348 in 2004; 0.156 in 2005; and 0.357 in 2006 .

\section{Costs}

The Infection Control Department was responsible for 450 inpatient beds in 3 buildings with 2.25 full-time equivalent (FTE) infection preventionists and a 0.6 FTE manager. The infection control manager adjusted workloads to provide for the creation, ongoing implementation, training, and dissemination of performance feedback by assigning a proportion of manual surveillance activities to automated systems and prioritizing hand hygiene as the educational priority. There were no additional costs for incentives: an existing "Awards and Recognition" hospital fund was diverted to pay for hand

TABLE 2. Rates of Hand Hygiene Compliance by Healthcare Worker (HCW) Category and Intervention Period Proportion of opportunities with compliance (\%)

\begin{tabular}{lrrrrrr}
\cline { 2 - 7 } HCW category & \multicolumn{5}{c}{ At baseline } & \multicolumn{1}{c}{ At initial } \\
intervention & \multicolumn{1}{c}{2003} & \multicolumn{1}{c}{2004} & \multicolumn{1}{c}{2005} & \multicolumn{1}{c}{2006} \\
\hline Physician & $139 / 516(27)$ & $389 / 823(47)$ & $579 / 919(63)$ & $468 / 768(61)$ & $412 / 722(57)$ & $348 / 580(60)$ \\
Nursing & $857 / 3,173(27)$ & $2,674 / 4,951(54)$ & $5,100 / 7,083(72)$ & $4,182 / 5,100(82)$ & $3,216 / 4,288(75)$ & $3205 / 4,273(75)$ \\
Other ancillary & $170 / 487(35)$ & $290 / 580(50)$ & $440 / 677(65)$ & $442 / 552(80)$ & $253 / 366(69)$ & $86 / 137(63)$ \\
\hline
\end{tabular}


TABLE 3. Survey Responses by Healthcare Worker Category regarding Attitudes toward and Practices of Hand Hygiene

\begin{tabular}{|c|c|c|c|}
\hline \multirow[b]{2}{*}{ Area assessed, survey question } & \multicolumn{2}{|c|}{$\begin{array}{c}\text { Proportion (\%) of } \\
\text { respondents answering Yes }\end{array}$} & \multirow[b]{2}{*}{$P^{a}$} \\
\hline & $\begin{array}{l}\text { Physician } \\
(n=54)\end{array}$ & $\begin{array}{c}\text { Nurse } \\
(n=115)\end{array}$ & \\
\hline \multicolumn{4}{|l|}{ Attitude } \\
\hline Is hand hygiene very important to reduce spread of resistant organisms? & $45 / 53(85)$ & $97 / 109(89)$ & .456 \\
\hline Can you improve your hand hygiene practices? & $24 / 51(47)$ & $57 / 97(59)$ & .224 \\
\hline \multicolumn{4}{|l|}{ Reinforcement } \\
\hline Have you ever been praised for good hand hygiene? & $29 / 54(54)$ & $88 / 109(81)$ & $<.001^{\mathrm{b}}$ \\
\hline Have you ever received a candy bar for good hand hygiene? & $29 / 54(54)$ & $83 / 109(76)$ & $<.001^{\mathrm{b}}$ \\
\hline \multicolumn{4}{|l|}{ Healthcare worker participation } \\
\hline Do you discuss hand hygiene with your coworkers at least weekly? & $17 / 54(31)$ & $80 / 105(61)$ & $<.001^{\mathrm{b}}$ \\
\hline Do you remind another $\mathrm{HCW}$ to perform hand hygiene at least weekly? & $12 / 54(22)$ & $33 / 113(29)$ & .456 \\
\hline Authority figure & & & \\
\hline Does your "boss" remind you to perform hand hygiene at least weekly? & $7 / 54(13)$ & $48 / 111(43)$ & $<.001^{\mathrm{b}}$ \\
\hline \multicolumn{4}{|l|}{ 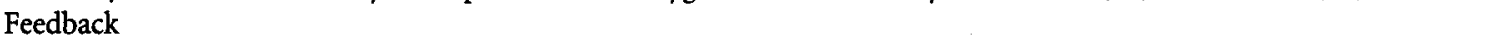 } \\
\hline Do you see hand hygiene rates every month? & $4 / 54(7)$ & $50 / 110(45)$ & $<.001^{\mathrm{b}}$ \\
\hline Do you see hand hygiene rates less than once a year? & $26 / 54(48)$ & $4 / 110(4)$ & $<.001^{b}$ \\
\hline
\end{tabular}

NOTE. Not all respondents answered every survey question; the proportion of those respondents answering Yes is expressed as a percentage.

a Differences in physician-to-nurse proportion of positive responses compared using Fisher's exact test.

b Statistically significant.

hygiene rewards amounting to $\$ 3,000$ per year for 5,000 candy bars, $\$ 700$ per year for pizza and other unit awards during years when motivating groups was prioritized, and $\$ 700$ per year for gift certificates during years when individuals were incentivized. New expenses included purchase of the alcohol sanitizer at a time before it was widely used in healthcare facilities. The product was donated during the initial campaign, and in subsequent years, the cost was approved by hospital administration. Additional expenses included collection and entry of hand hygiene observations by several parttime hand hygiene monitors. Hospital administration supported a 0.35 FTE clerk salary at $\$ 10,000$ per year to compensate part-time staff who collectively worked a total of 14 hours per week.

\section{DISCUSSION}

Our hand hygiene promotion program successfully implemented a sophisticated behavioral change approach and is one of the earliest and largest such programs reported from the United States. The World Health Organization advocates such institutional culture-changing hand hygiene programs to improve patient safety. Most successful large programs have come from the international community, beginning with a hospital-wide sustained hand hygiene improvement campaign in Europe,' to multihospital programs effective in maintaining improved hand hygiene across Australia ${ }^{29}$ and at National Health Services Hospitals in the United Kingdom. ${ }^{30}$ All of these campaigns were multidimensional, heavily promoted, evaluated all "5 moments for hand hygiene" (as outlined by the World Health Organization), ${ }^{31}$ and were associated with decreases in MRSA infections. Similar levels of success have not been reported from the United States. One multimodal program in several Chicago hospitals led to modest improvements in the hand hygiene compliance rate. ${ }^{32} \mathrm{~A}$ survey of 40 National Healthcare Safety Network hospitals reported low hand hygiene compliance rates, despite the presence of policies consistent with the Centers for Disease Control and Prevention hand hygiene guidelines; although most staff were aware of the guidelines and had access to alcohol sanitizer product, multidisciplinary promotion activities were lacking in almost half of these hospitals. ${ }^{33}$

Our program was multidimensional. Key components that other programs have also used included an educational blitz, ready access to and adoption of alcohol sanitizer, frequent feedback regarding hand hygiene performance, and administrative support. Other aspects of our program that are less commonly employed by other programs included "grassroots" involvement from individual nursing units and a special emphasis on behavior modification. A unique feature was our immediate positive reinforcement given to those "caught in the act" of performing hand hygiene. Because "automatic," ingrained hand hygiene behaviors are difficult to change, ${ }^{22}$ we used operant conditioning with positive reinforcement to "hardwire in" desired responses. ${ }^{34,35}$ Bestowing incentives when peers were present enhanced the impact for compliant individuals, whereas noncompliant HCWs "got the message" nonconfrontationally. Striking a balance between providing positive reinforcement and holding HCWs with repeated violations accountable is likely to be the next step to optimal patient safety. ${ }^{36}$

Although significant and sustained increases in hand hygiene compliance rates among physicians were seen, gains 
were not as great among physicians as they were among other HCWs. Our survey suggests that physicians had less exposure to program interventions, including positive rewards and feedback, than did nurses and other HCWs. We found it more difficult to reach physicians because they function more independently than other HCWs, change rotations frequently, and tend to care for patients in multiple locations. Additional efforts have been directed at identifying physician hand hygiene "champions" and encouraging mentors, assessment of hand hygiene by the lead physician during team work rounds, and developing incentives targeted for physicians.

Although there was a decreasing trend in the incident rate of healthcare-associated MRSA infection, neither MRSA nor VRE infection rates changed significantly during the hand hygiene program, despite substantial increases in hand hygiene compliance rates. This may be attributable to other factors, including the importance of compliance with all 5 moments for hand hygiene and adherence to contact precautions for patients with infection due to a multidrug-resistant organism.

Several limitations of this study merit consideration. Standardization and optimal measurement of hand hygiene observations is always a concern. Although our observers remained unobtrusive, we could not eliminate a Hawthorne effect, which has been shown to influence hand hygiene compliance. ${ }^{37}$ We recognize that our hand hygiene compliance data may be inflated; we used a best-case scenario. Nevertheless, this method was applied consistently across the hospital by trained external observers and was useful to monitor trends over the course of the program. Another limitation is contamination of the control units, evidenced by the almost $7 \%$ alcohol sanitizer use among baseline observations.

Our comprehensive campaign continues, with the goal being to maintain hand hygiene compliance: there are always new staff, and ongoing feedback and reinforcement remain important for all HCWs. Overall, we still observe good hand hygiene compliance; compliance rates before and after patient contact remain similar to rates reported at the end of this study. However, subsequent observations of hand hygiene done during patient care activities have shown the rate of adherence to be less than that before or after patient contact. This has been a lesson learned, and although more resources will be needed, our institution feels that it is important to develop a more comprehensive and staff-led monitoring program. We are now focusing efforts on improving compliance with all indications for hand hygiene using the WHO " 5 moments for hand hygiene," ${ }^{, 31}$ as recently described in reports from several international programs. ${ }^{29,30}$

In conclusion, our large-scale interventional cohort study used a culture-change approach and is, to our knowledge, the largest and most prolonged institution-wide program to promote alcohol sanitizer in the United States. We implemented this creative campaign using ongoing frequent audit and feedback with a novel use of immediate positive reinforcement at an acceptable cost to the institution. An im- portant feature of the program was the collaboration and teamwork between infection control personnel and individual unit managers and staff. Significant and sustained improvements in hand hygiene were realized in all HCW categories, including among physicians. Future goals will aim to improve compliance with all indications for hand hygiene, as well as to reduce the number of healthcare-associated infections.

\section{ACKNOWLEDGMENTS}

We are grateful to the Thatcher Company (Salt Lake City, UT) for donating alcohol sanitizer product during the initial intervention campaign, and to Marianne Madsen and Janelle Howell for their helpful review of the manuscript.

Potential conflicts of interest. All authors report no conflicts of interest relevant to this article.

Address reprint requests to Jeanmarie Mayer, MD, Division of Epidemiology, University of Utah, 295 Chipeta Way, Salt Lake City, UT 84132 (jeanmarie.mayer@hsc.utah.edu).

\section{REFERENCES}

1. Pittet D, Hugonnet S, Harbarth S, et al. Effectiveness of a hospital-wide programme to improve compliance with hand hygiene. Lancet 2000;356: $1307-1312$.

2. Won SP, Chou HC, Hsieh WS, et al. Handwashing program for the prevention of nosocomial infections in a neonatal intensive care unit. Infect Control Hosp Epidemiol 2004;25:742-746.

3. Rosenthal VD, Guzman S, Safdar N. Reduction in nosocomial infection with improved hand hygiene in intensive care units of a tertiary care hospital in Argentina. Am J Infect Control 2005;33(7):392-397.

4. Pittet D, Simon A, Hugonnet S, et al. Hand hygiene among physicians: performance, beliefs, and perceptions. Ann Intern Med 2004;141:1-8.

5. Kennedy AM, Elward AM, Fraser VJ. Survey of knowledge, beliefs, and practices of neonatal intensive care unit healthcare workers regarding nosocomial infections, cental venous catheter care, and hand hygiene. Infect Control Hosp Epidemiol 2004;25:747-752.

6. Boyce IM, Pittet D. Guideline for hand hygiene in health-care settings: Recommendations of the Healthcare Infection Control Practices Advisory Committee and the HICPAC/SHEA/APIC/IDSA Hand Hygiene Task Force. MMWR Morb Mortal Wkly Rep 2002;51(RR-16):1-45.

7. Larson EL. APIC guideline for handwashing and hand antisepsis in health care settings. Am J Infect Control 1995;23:251-269.

8. Association of Operating Room Nurses Recommended Practices Committee. Recommended practices: surgical hand scrubs. AORN I 1990;52: 830-836.

9. World Health Organization (WHO). World Alliance for Patient Safety. Global Patient Safety Challenge 2005-2006. Clean care is safer care. Geneva: WHO, 2005. http://www.who.int/patientsafety/events/05/GPSC_Launch _ENGLISH_FINAL.pdf. Accessed December 1, 2010.

10. Grol R, Grimshaw J. From best evidence to best practice: effective implementation of change in patients' care. Lancet 2003;362:1225-1230.

11. Erasmus V, Daja TJ, Brug H, et al. Systematic review of studies on compliance with hand hygiene guidelines in hospital care. Infect Control Hosp Epidemiol 2010;31:283-294.

12. Voss A, Widmer AF. No time for handwashing!? Handwashing versus alcoholic rub: can we afford 100\% compliance? Infect Control Hosp Epidemiol 1997;18:205-208.

13. Pittet $D$, Mourouga $P$, Perneger TV. Compliance with handwashing in a teaching hospital. Ann Intern Med 1999;130:126-130.

14. Bischoff WE, Reynolds TM, Sessler $\mathrm{CN}$, et al. Handwashing compliance by health care workers. Arch Intern Med 2000;160:1017-1021.

15. Maury E, Alzieu M, Baudel JL, et al. Availability of an alcohol solution 
can improve hand disinfection compliance in an intensive care unit. Am J Respir Crit Care Med 2000;162:324-327.

16. Naikoba $S$, Hayward A. The effectiveness of interventions aimed at increasing handwashing in healthcare workers-a systematic review. J Hosp Infect 2001;47(3):173-180.

17. Cockburn J. Adoption of evidence into practice: can change be sustainable? MJA 2004;180:S66-S67.

18. Moulding NT, Silagy CA, Weller DP. A framework for effective management of change in clinical practice: dissemination and implementation of clinical practice guidelines. Quality in Health Care 1999;8:177183.

19. Bero LA, Grilli R, Grimshaw JM, et al. Closing the gap between research and practice: an overview of systematic reviews of interventions to promote the implementation of research findings. BMJ 1998;317:465-468.

20. Sutton S. Health behavior: psychosocial theories. International encyclopedia of the social and behavioral sciences. Smelser NJ, Baltes PB, eds. Oxford, UK: Elsevier, 2001.

21. O'Boyle CA, Henly SI, Larson E. Understanding adherence to hand hygiene recommendations: the theory of planned behavior. Am J Infect Control 2001;29:352-360.

22. Whitby M, McLaws ML, Ross MW. Why healthcare workers don't wash their hands: a behavioral explanation. Infect Control Hosp Epidemiol 2006;27:484-492.

23. Raboud J, Saskin R, Wong $\mathrm{K}$, et al. Patterns of handwashing behavior and visits to patients on a general medical ward of healthcare workers. Infect Control Hosp Epidemiol 2004;25:198-202.

24. . Alvaran MS, Butz A, Larson E. Opinions, knowledge, and self reported practices related to infection control among nursing personnel in longterm care settings. Am J Infect Control 1994;22:367-370.

25. Giuffrida A, Torgerson DJ. Should we pay the patient? Review of financial incentives to enhance patient compliance. BMJ 1997;315:703-707.

26. Leitenberg H, Agras WS, Thomson LE. A sequential analysis of the effect of selective positive reinforcement in modifying anorexia nervosa. Behav Res Ther 1968;6:211-218.

27. Hand Hygiene SuperStar Nomination Form at the Johns Hopkins Hos- pital. http://www.hopkinsmedicine.org/heic/Hand_Hygiene/. Accessed December 1, 2010.

28. Carroll CM, Gladson SW, Camins BC. "Your hands touch many lives": a hand hygiene compliance improvement campaign [abstract]. Am J Infect Control 2006;34(5):E27-E28.

29. Grayson ML, Jarvie LJ, Martin R, et al. Significant reductions in methicillin-resistant Staphylococcus aureus bacteraemia and clinical isolates associated with a multisite, hand hygiene culture-change program and subsequent statewide roll-out. Med J Aust 2008;188(11):633-640.

30. Stone SP, Fuller C, Slade R, et al. National observational study of the effectiveness of the Clean Your Hands Campaign (NOSEC) July 2004Dec 2008: each extra $\mathrm{mL}$ alcohol handrub per patient-bed-day associated with a $1 \%$ drop in MRSA bacteraemia [abstract]. In: Program and abstracts of the ANnual Scientific Meeting of the Society for Healthcare Epidemiology of America; March, 2009; San Diego, CA.

31. World Health Organization (WHO). Five moments for hand hygiene. Geneva, Switzerland: WHO, 2009.http://www.who.int/gpsc/tools/Five moments/en/index.html Accessed April 2009.

32. Trick WE, Vernon MO, Welbel SO, et al. Multicenter intervention program to increase adherence to hand hygiene recommendations and glove use and to reduce the incidence of antimicrobial resistance. Infect Control Hosp Epidemiol 2007;28:42-49.

33. Larson EL, Quiros D, Lin SX. Dissemination of the CDC's hand hygiene guideline and impact on infection rates. Am J Infect Control 2007;35(10): 666-675.

34. Blackman D. Operant conditioning: an experimental analysis of behaviour. London, UK: Methuen, 1974.

35. Ouellette JA, Wood W. Habit and intention in everyday life: the multiple processes by which past behaviour predicts future behaviour. Psychol Bull 1998;124:54-74.

36. Goldman D. System failure versus personal accountability-the case for clean hands. $N$ Engl J Med 2006;355:121-123.

37. Eckmanns T, Bessert J, Behnke, et al. Compliance with antiseptic hand rub use in intensive care units: the Hawthorne effect. Infect Control Hosp Epidemiol 2006;27(9):931-934. 